\title{
$A$-Statistical Cluster Points in Finite Dimensional Spaces and Application to Turnpike Theorem
}

\author{
Pratulananda Das, ${ }^{1}$ Sudipta Dutta, ${ }^{1}$ S. A. Mohiuddine, ${ }^{2}$ and Abdullah Alotaibi ${ }^{2}$ \\ ${ }^{1}$ Department of Mathematics, Jadavpur University, Kolkata West Bengal, 700032, India \\ ${ }^{2}$ Department of Mathematics, Faculty of Science, King Abdulaziz University, P.O. Box 80203, Jeddah 21589, Saudi Arabia \\ Correspondence should be addressed to S. A. Mohiuddine; mohiuddine@gmail.com
}

Received 6 August 2013; Accepted 19 December 2013; Published 28 January 2014

Academic Editor: Andrew Pickering

Copyright @ 2014 Pratulananda Das et al. This is an open access article distributed under the Creative Commons Attribution License, which permits unrestricted use, distribution, and reproduction in any medium, provided the original work is properly cited.

In the first part of the paper, following the works of Pehlivan et al. (2004), we study the set of all $A$-statistical cluster points of sequences in $m$-dimensional spaces and make certain investigations on the set of all $A$-statistical cluster points of sequences in $m$-dimensional spaces. In the second part of the paper, we apply this notion to study an asymptotic behaviour of optimal paths and optimal controls in the problem of optimal control in discrete time and prove a general version of turnpike theorem in line of the work of Mamedov and Pehlivan (2000). However, all results of this section are presented in terms of a more general notion of $\mathscr{I}$-cluster points.

\section{Introduction and Background}

Throughout this paper, let $A$ be a nonnegative regular matrix and $\mathbb{N}$ will denote the set of all positive integers. Let $X$ and $Y$ be two sequence spaces and $A=\left(a_{n k}\right)$ be an infinite matrix. If for each $x \in X$ the series $A_{n}(x)=\sum_{k=1}^{\infty} a_{n k} x_{k}$ converges for each $n$ and the sequence $A x=\left\{A_{n}(x)\right\} \in Y$, we say that A maps $X$ into $Y$. By $(X, Y)$ we denote the set of all matrices which maps $X$ into $Y$. In addition if the limit is preserved, then we denote the class of such matrices by $(X, Y)_{\text {reg. }}$ A matrix $A$ is called regular if $A \in(c, c)$ and $\lim _{k \rightarrow \infty} A_{k}(x)=$ $\lim _{k \rightarrow \infty} x_{k}$ for all $x=\left\{x_{k}\right\}_{k \in \mathbb{N}} \in c$ when $c$, as usual, stands for the set of all convergent sequences. It is well known that the necessary and sufficient condition for $A$ to be regular are
(a) $\|A\|=\sup _{n} \sum_{k}\left|a_{n k}\right|<\infty$;
(b) $\lim _{n} a_{n k}=0$, for each $k$;
(c) $\lim _{n} \sum_{k} a_{n k}=1$.

The idea of $A$-statistical convergence was introduced by Kolk [1] using a nonnegative regular matrix $A$. For a nonnegative regular matrix $A=\left(a_{n k}\right)$, a set $K \subset \mathbb{N}$ will be said to have $A$-density if $\delta_{A}(K):=\lim _{n} \sum_{k \in K} a_{n k}$ exists. The real number sequence $x=\left\{x_{k}\right\}_{k \in \mathbb{N}}$ is said to be $A$-statistically convergent to $L$ provided that for every $\varepsilon>0$ the set $K(\varepsilon):=$ $\left\{k \in \mathbb{N}:\left|x_{k}-L\right| \geq \varepsilon\right\}$ has $A$-density zero. Note that the idea of $A$-statistical convergence is an extension of the idea of statistical convergence introduced by Fast [2] using the idea of asymptotic density and later studied by Fridy [3, 4], Connor [5], and Šalát [6] (see also [1, 7-11] for more references). Let $K=\{k(j): k(1)<k(2)<k(3)<\cdots\} \subset \mathbb{N}$ and $\{x\}_{K}=\left\{x_{k(j)}\right\}$ be a subsequence of $x$. If the set $K$ has $A$-density zero then the subsequence $\{x\}_{K}$ of the sequence $x$ is called an $A$-thin subsequence. If the set $K$ does not have $A$-density zero then the subsequence $\{x\}_{K}$ is called an $A$-nonthin subsequence of $x$. The statement $\delta_{A}(K) \neq 0$ means that either $\delta_{A}(K)>0$ or $\delta_{A}(K)$ does not exist.

A family $\mathscr{I} \subset 2^{Y}$ of subsets of a nonempty set $Y$ is said to be an ideal in $Y$ if (i) $A, B \in \mathscr{I}$ imply $A \cup B \in \mathscr{I}$; (ii) $A \in \mathscr{I}, B \subset A$ implies $B \in \mathscr{I}$, while an admissible ideal $\mathscr{I}$ of $Y$ further satisfies $\{x\} \in \mathscr{I}$ for each $x \in Y$. If $\mathscr{I}$ is a proper ideal in $Y$ (i.e, $Y \notin \mathscr{I}, Y \neq \emptyset$ ), then the family of sets $F(\mathscr{I})=\{M \subset Y:$ there exists $A \in \mathscr{I}: M=Y \backslash A\}$ is a filter in $Y$. It is called the filter associated with the ideal $\mathscr{I}$. Throughout $\mathscr{I}$ will stand for a proper nontrivial admissible ideal of $\mathbb{N}$. Let $K=\{k(j): k(1)<k(2)<k(3)<\cdots\} \subset \mathbb{N}$ and 
$\{x\}_{K}=\left\{x_{k(j)}\right\}$ be a subsequence of $x$. If the set $K$ belongs to $\mathscr{I}$, then the subsequence $\{x\}_{K}$ of the sequence $x$ is called a $\mathscr{I}$ thin subsequence and if the set $K$ does not belong to $\mathscr{I}$, then the subsequence $\{x\}_{K}$ is called a $\mathscr{I}$-nonthin subsequence of $x$.

In this context, it should be mentioned that ideals were first used to generalize the idea of statistical convergence by Kostyrko et al. [12]. For more recent applications of ideals one can see $[1,4,13-21]$ where many more references can be found.

The concept of statistical cluster points was introduced by Fridy [4]. A real number $\gamma$ is a statistical cluster point of $x$ if for every $\varepsilon>0$; the set $\left\{k:\left|x_{k}-\gamma\right|<\varepsilon\right\}$ does not have asymptotic density zero. It was shown that the set of all statistical cluster points is nonempty and compact. Later the notion of statistical cluster points was extended to $\mathscr{I}$-cluster points in [12] (see also [9]) and to $A$-statistical cluster point by Demirci [22] (see also [23]) and very recently to $\lambda$-statistical cluster point by Pehlivan et al. [24]. A number $\gamma$ is said to be an $A$-statistical cluster point of the number sequence $x=$ $\left\{x_{k}\right\}_{k \in \mathbb{N}}$ provided that for every $\varepsilon>0, \delta_{A}\left(K_{\varepsilon}\right) \neq 0$, where $K_{\varepsilon}:=\left\{k \in \mathbb{N}:\left|x_{k}-\gamma\right|<\varepsilon\right\}$. By $\Gamma_{A}(x)$, we denote the set of all $A$-statistical cluster points of $x$. Many interesting results concerning statistical and then $\mathscr{I}$-cluster points were proved in [8] and then in [25].

In this paper, following the line of [26], we first investigate certain properties of the set of $A$-statistical cluster points in $\mathbb{R}^{m}$ and the concept of $\Gamma^{A}$-statistical convergence and examine some of its consequences. Though some of our results were proved in [25] for arbitrary ideals of $\mathbb{N}$, we present the results for a specific ideal consisting of $A$-density zero sets with alternative methods of proofs. Finally, we present an application by establishing a general version of turnpike theorem in line of the results proved by Pehlivan and Mamedov [27, 28], which appears to be valid for a special class of ideals.

\section{Characterization of $\Gamma_{A}(x)$}

In this section, we investigate some properties of the set of all $A$-statistical cluster points in $\mathbb{R}^{m}$ with usual norm $\|\cdot\|$. Consider a sequence $x=\left\{x_{k}\right\}_{k \in \mathbb{N}}, x_{k} \in \mathbb{R}^{m}$ and a point $\zeta \in$ $\mathbb{R}^{m}$. Following [1], we consider the following definitions.

Definition 1. A sequence $x=\left\{x_{k}\right\}_{k \in \mathbb{N}}$ is $A$-statistically convergent to $\zeta$ if for every $\varepsilon>0$

$$
\delta_{A}\left\{k \in \mathbb{N}:\left\|x_{k}-\zeta\right\| \geq \varepsilon\right\}=0 .
$$

Definition 2. A point $\zeta$ is called an $A$-statistical cluster point of the sequence $x=\left\{x_{k}\right\}_{k \in \mathbb{N}}$ if for every $\varepsilon>0$

$$
\delta_{A}\left\{k \in \mathbb{N}:\left\|x_{k}-\zeta\right\|<\varepsilon\right\} \neq 0 .
$$

We denote the set of all $A$-statistical cluster points of the sequence $x=\left\{x_{k}\right\}_{k \in \mathbb{N}}$ by $\Gamma_{A}(x)$. Now from Definition 2 it readily follows that

$$
\lim _{n} \sum_{k \in K_{\varepsilon}} a_{n k} \neq 0
$$

where

$$
K_{\varepsilon}:=\left\{k \in \mathbb{N}:\left\|x_{k}-\zeta\right\|<\varepsilon\right\} .
$$

That is,

$$
\lim _{n} \sum_{k=1}^{\infty} a_{n k} \chi_{K_{\varepsilon}}(k) \neq 0 .
$$

Hence,

$$
\lim _{n} \sup \sum_{k=1}^{\infty} a_{n k} \chi_{K_{\varepsilon}}(k)>0 \text {. }
$$

Definition 3. A sequence $x=\left\{x_{k}\right\}_{k \in \mathbb{N}}$ is said to be $A$-statistically bounded if there exists a compact set $B$ such that $\delta_{A}\left\{k: x_{k} \notin B\right\}=0$.

The above definitions can be generalized via ideals as follows.

Definition 4 (see $[9,12])$. A sequence $x=\left\{x_{k}\right\}_{k \in \mathbb{N}}$ is $\mathscr{I}$ convergent to $\zeta$ if for any $\varepsilon>0,\left\{k \in \mathbb{N}:\left\|x_{k}-\zeta\right\| \geq \varepsilon\right\} \in \mathscr{I}$.

Definition 5 (see $[9,12])$. A point $\zeta$ is called an $\mathscr{I}$-cluster point of the sequence $x=\left\{x_{k}\right\}_{k \in \mathbb{N}}$ if for every $\varepsilon>0$

$$
\left\{k \in \mathbb{N}:\left\|x_{k}-\zeta\right\|<\varepsilon\right\} \notin \mathscr{I} .
$$

We denote the set of all $\mathscr{I}$-cluster points by $\Gamma_{\mathscr{I}}(x)$.

Definition 6. A sequence $x=\left\{x_{k}\right\}_{k \in \mathbb{N}}$ is said to be $\mathscr{I}$ bounded if there exists a compact set $B$ such that $\left\{k: x_{k} \notin\right.$ $B\} \in \mathscr{I}$.

Let $d(C, \xi)$ stand for the distance of a point $\xi$ from a closed set $C$ and it is defined as $d(C, \xi)=\inf _{y \in C}\|y-\xi\|$. Let $S_{\varepsilon}(C)=$ $\left\{y \in \mathbb{R}^{m}: d(C, y)<\varepsilon\right\}$ be the open $\varepsilon$-neighborhood of $C$.

Lemma 7. Let $K$ be a compact subset of $\mathbb{R}^{m}$ such that $K \cap$ $\Gamma_{A}(x)=\emptyset$. Then $\delta_{A}\left\{k: x_{k} \in K\right\}=0$.

In [8, Lemma 2.1], Činčura et al. proved the ideal version of the above lemma which also extends Lemma 1 [26].

In [26], it was shown that for the ideal $\mathscr{I}_{d}$ of density zero sets, the above result is not true for open or unbounded subsets of $\mathbb{R}^{m}$; that is, the assumption of compactness is essential. However, in [8], no such example was presented to show the essentiality of compactness. Below we present two examples which show that the assumption of compactness is essential even if we take the ideal of subsets of $\mathbb{N}$ with $A$ density zero.

Next we show that the compactness of $K$ is essential for Lemma 7. The result may fail if one of closedness or boundedness hypotheses is relaxed. 
Remark 8 . Let $K$ be the unbounded closed set $[1, \infty)$. Consider the following nonnegative regular matrix:

$$
A=\left(\begin{array}{ccccccccc}
\frac{1}{2^{2}} & \frac{1}{2^{2}} & 0 & 0 & 0 & 0 & 0 & 0 & \cdots \\
\frac{1}{2^{3}} & \frac{1}{2^{3}} & \frac{1}{2^{2}} & \frac{1}{2^{2}} & 0 & 0 & 0 & 0 & \cdots \\
\frac{1}{2^{4}} & \frac{1}{2^{4}} & \frac{1}{2^{3}} & \frac{1}{2^{3}} & \frac{1}{2^{2}} & \frac{1}{2^{2}} & 0 & 0 & \cdots \\
\cdot & \cdot & \cdot & \cdot & \cdot & \cdot & . & . & \cdots \\
\cdot & \cdot & . & . & . & . & . & . & \cdots \\
\cdot & \cdot & \cdot & . & . & . & . & . & \cdots
\end{array}\right)
$$

Now for the sequence $x=\{0,1,0,2,0,3, \ldots\}, \Gamma_{A}(x)=\{0\}$ as $\delta_{A}\left\{k:\left\|x_{k}-0\right\|<\varepsilon\right\}=1 / 2 \neq 0$. In this case, we have $K \cap \Gamma_{A}(x)=$ $\emptyset$ but $\delta_{A}\left\{k: x_{k} \in K\right\}=\delta_{A}\{2,4,6, \ldots\}=1 / 2 \neq 0$.

Remark 9. Let $K$ be the bounded open set $(0,1)$. Consider the nonnegative regular matrix:

$$
A=\left(\begin{array}{cccccccc}
\frac{1}{2} & 0 & 0 & 0 & 0 & 0 & 0 & \cdots \\
\frac{1}{2^{2}} & 0 & \frac{1}{2} & 0 & 0 & 0 & 0 & \cdots \\
\frac{1}{2^{3}} & 0 & \frac{1}{2^{2}} & 0 & \frac{1}{2} & 0 & 0 & \cdots \\
\frac{1}{2^{4}} & 0 & \frac{1}{2^{3}} & 0 & \frac{1}{2^{2}} & 0 & \frac{1}{2} & \cdots \\
\cdot & \cdot & \cdot & . & \cdot & . & \cdot & . \\
\cdot & \cdot & \cdot & . & . & . & . & . \\
\cdot & \cdot & \cdot & . & . & . & . & .
\end{array}\right) .
$$

Clearly for the sequence $x=\{1 / k\}_{k \in \mathbb{N}}$, we have $\Gamma_{A}(x)=\{0\}$. In this case also, we have $K \cap \Gamma_{A}(x)=\emptyset$, but we have $\delta_{A}\{k$ : $\left.x_{k} \in K\right\}=\delta_{A}\{2,3,4, \ldots\}=1 \neq 0$.

Lemma 10. If a sequence $x=\left\{x_{k}\right\}_{k \in \mathbb{N}}$ has a bounded $A$ nonthin subsequence then the set $\Gamma_{A}(x)$ is nonempty and closed.

Lemma 11. If $x=\left\{x_{k}\right\}_{k \in \mathbb{N}}$ is A-statistically bounded, then the set $\Gamma_{A}(x)$ is nonempty and compact.

Letavaj [25] proved the ideal version of the above lemma. Next we prove the following interesting theorem.

Theorem 12. If $x=\left\{x_{k}\right\}_{k \in \mathbb{N}}$ is an A-statistically bounded sequence then for every $\varepsilon>0$,

$$
\delta_{A}\left\{k: d\left(\Gamma_{A}(x), x_{k}\right) \geq \varepsilon\right\}=0 .
$$

Proof. Since $x$ is an $A$-statistically bounded sequence, there exists a compact set $B$ such that $\delta_{A}\left\{k: x_{k} \notin B\right\}=0$. Now $\Gamma_{A}(x)$ is nonempty and $\Gamma_{A}(x) \subset B$ by Lemma 11. Suppose that $\delta_{A}\left\{k: d\left(\Gamma_{A}(x), x_{k}\right) \geq \varepsilon\right\} \neq 0$. Then there exists a number $\varepsilon>$ 0 , such that $\lim _{n} \sup \sum_{k=1}^{\infty} a_{n k} \chi_{K_{\varepsilon}}(k)>0$, where $K_{\varepsilon}:=\{k:$ $\left.d\left(\Gamma_{A}(x), x_{k}\right) \geq \varepsilon\right\}$. Define $S_{\varepsilon}\left(\Gamma_{A}(x)\right)=\left\{y: d\left(\Gamma_{A}(x), y\right)<\right.$ $\varepsilon\}$ and let $D=B \backslash S_{\varepsilon}\left(\Gamma_{A}(x)\right)$. Consequently, $D$ is compact and contains an $A$-nonthin subsequence of $x$. Hence, from Lemma 7 it follows that $D \cap \Gamma_{A}(x) \neq \emptyset$. Therefore $D$ contains an $A$-statistical cluster point which is a contradiction. This proves the result.
Remark 13. If we take the sequence $x=\{1,0,2,0,3,0, \ldots\}$ and consider the matrix $A$ given in Remark 8 , then we get $\Gamma_{A}(x)=\{0\}$, but $\delta_{A}\left\{k: d\left(\Gamma_{A}(x), x_{k}\right) \geq \varepsilon\right\}=1 / 2 \neq 0$. Hence, we can conclude that if the sequence $x$ is not bounded then Theorem 12 need not be true.

\section{3. $\Gamma^{A}$-Statistical Convergence to a Set}

Definition 14. Let $C \subset \mathbb{R}^{m}$ be a closed set satisfying the property

$$
\delta_{A}\left\{k: d\left(C, x_{k}\right) \geq \varepsilon\right\}=0 \quad \text { for every } \varepsilon>0 .
$$

Then set $C$ is said to be an $A$-minimal closed set if for every closed set $C^{\prime} \subset C\left(C \backslash C^{\prime} \neq \emptyset\right)$, there exists a number $\varepsilon^{\prime}>0$ such that

$$
\delta_{A}\left\{k: d\left(C^{\prime}, x_{k}\right) \geq \varepsilon^{\prime}\right\} \neq 0 .
$$

Definition 15. A sequence $x=\left\{x_{k}\right\}_{k \in \mathbb{N}}$ is said to be $\Gamma^{A}$ statistically convergent to the set $C$ if $C$ is a nonempty $A$ minimal closed set.

Generalizing the above concepts using ideals we can have the following defintion.

Definition 16. Let $C \subset \mathbb{R}^{m}$ be a closed set satisfying the property

$$
\left\{k: d\left(C, x_{k}\right) \geq \varepsilon\right\} \in \mathscr{I}, \quad \text { for every } \varepsilon>0 .
$$

Then set $C$ is said to be an $\mathscr{I}$-minimal closed set if for every closed set $C^{\prime} \subset C\left(C \backslash C^{\prime} \neq \emptyset\right)$, there exists a number $\varepsilon^{\prime}>0$ such that

$$
\left\{k: d\left(C^{\prime}, x_{k}\right) \geq \varepsilon^{\prime}\right\} \notin \mathscr{I} .
$$

A sequence $x=\left\{x_{k}\right\}_{k \in \mathbb{N}}$ is called $\Gamma^{\mathscr{I}}$-convergent to the set $C$ if $C$ is a nonempty $\mathscr{I}$-minimal closed set.

Following the line of Theorem 4 in [26], we now give the following theorem.

Theorem 17. If $x=\left\{x_{k}\right\}_{k \in \mathbb{N}}$ is A-statistically bounded then it is $\Gamma^{A}$-statistically convergent to the set $\Gamma_{A}(x)$.

Proof. From Lemma 11 and Theorem $12, \Gamma_{A}(x)$ is a nonempty compact set and $\delta_{A}\left\{k: d\left(\Gamma_{A}(x), x_{k}\right) \geq \varepsilon\right\}=0$, for every $\varepsilon>0$. We need to show that $\Gamma_{A}(x)$ is an $A$-minimal set.

On the contrary, suppose that $\Gamma_{A}(x)$ is not $A$-minimal. Then there exists a closed set $C \subset \Gamma_{A}(x)\left(\Gamma_{A}(x) \backslash C \neq \emptyset\right)$ with $\delta_{A}\left\{k: d\left(C, x_{k}\right) \geq \varepsilon\right\}=0$. Hence, there is a point $\zeta \in \Gamma_{A}(x)$ such that $\zeta \notin C$ and so there exists a number $\varepsilon>0$ such that $S_{\varepsilon}(\zeta) \cap S_{\varepsilon}(C)=\emptyset$. Now since $\zeta$ is an $A$-statistical cluster point so $\lim _{n} \sup \sum_{k \in K_{\varepsilon(\zeta)}} a_{n k} \chi_{K_{\varepsilon(\zeta)}}(k)>0$, where $K_{\varepsilon(\zeta)}:=\{k$ : $\left.d\left(\Gamma_{A}(x), x_{k}\right) \geq \varepsilon\right\}$. Since $S_{\varepsilon}(\zeta) \cap S_{\varepsilon}(C)=\emptyset$,

$$
\left\{k: x_{k} \in S_{\varepsilon}(\zeta)\right\} \subset\left\{k: x_{k} \notin S_{\varepsilon}(C)\right\} .
$$

Therefore $\lim _{n} \sup \sum_{k \in K_{\varepsilon(C)}} a_{n k} \chi_{K_{\varepsilon(C)}}(k)>0$, where $K_{\varepsilon(C)}:=$ $\left\{k: x_{k} \notin S_{\varepsilon}(C)\right\}$ which is a contradiction. This completes the proof. 
Remark 18. Define the sequence $x$ by

$$
x_{k}=p, \quad \text { where } k=2^{p-1}(2 q+1) ;
$$

that is, $p-1$ is the number of factors of 2 in the prime factorization of $k$. If we consider the matrix $A$ given in Remark 8, then we can show that the sequence is not $A$ statistically bounded. Here $\Gamma_{A}(x)=\{1,2,3, \ldots\}$ and the sequence $x$ is $\Gamma^{A}$-statistically convergent to $\Gamma_{A}(x)$.

Next we study the uniqueness of the $A$-limit set. We prove the following theorem in line of Theorem 5 in [26].

Theorem 19. If $x$ is $\Gamma^{A}$-statistically convergent to the set $C$, then $C=\Gamma_{A}(x)$.

Proof. We show that $\Gamma_{A}(x) \subset C$. On the contrary, let there be a point $\zeta \in \Gamma_{A}(x)$ such that $\zeta \notin C$. Now closedness of $C$ implies that there exists a number $\varepsilon>0$ for which $S_{\varepsilon}(\zeta) \cap S_{\varepsilon}(C)=\emptyset$ which consequently implies that

$$
\left\{k: x_{k} \in S_{\varepsilon}(\zeta)\right\} \subset\left\{k: x_{k} \notin S_{\varepsilon}(C)\right\} .
$$

Now since $\delta_{A}\left\{k: x_{k} \notin S_{\varepsilon}(C)\right\}=0$, so we get $\delta_{A}\left\{k: x_{k} \in\right.$ $\left.S_{\varepsilon}(\zeta)\right\}=0$. This implies that $\zeta \in \Gamma_{A}(x)$, a contradiction. Hence, $\Gamma_{A}(x) \subset C$.

Now we show that $C \subset \Gamma_{A}(x)$. Suppose $\zeta \in C$, but $\zeta \notin$ $\Gamma_{A}(x)$. Then there is a number $\varepsilon^{\prime}>0$ such that $\delta_{A}\left\{k: x_{k} \in\right.$ $\left.S_{\varepsilon}(\zeta)\right\}=0$ for every $\varepsilon \leq \varepsilon^{\prime}$. Now point $\zeta$ may be an isolated point or a limit point of $C$. So we consider the following cases.

Case 1. Let $\zeta$ be an isolated point $C$. Then there is a number $\varepsilon \leq \varepsilon^{\prime}$ such that $S_{\varepsilon}(\zeta) \cap S_{\varepsilon}(C \backslash\{\zeta\})=\emptyset$. This implies that $S_{\varepsilon}(C)=S_{\varepsilon}(\zeta) \cup S_{\varepsilon}(C \backslash\{\zeta\})$. Hence,

$$
\left\{k: x_{k} \notin S_{\varepsilon}(C \backslash\{\zeta\})\right\}=\left\{k: x_{k} \in S_{\varepsilon}(\zeta)\right\} \cup\left\{k: x_{k} \notin S_{\varepsilon}(C)\right\} .
$$

Therefore

$$
\begin{aligned}
\lim _{n} \sum_{k \in P} a_{n k} \chi_{P}(k)= & \lim _{n} \sum_{k \in \mathrm{Q}} a_{n k} \chi_{\mathrm{Q}}(k) \\
& +\lim _{n} \sum_{k \in R} a_{n k} \chi_{R}(k),
\end{aligned}
$$

where $P=\left\{k: x_{k} \notin S_{\varepsilon}(C \backslash\{\zeta\})\right\}, Q=\left\{k: x_{k} \in S_{\varepsilon}(\zeta)\right\}$ and $R=\left\{k: x_{k} \notin S_{\varepsilon}(C)\right\}$. It now readily follows that $\lim _{n} \sum_{k \in P} a_{n k} \chi_{P}(k)=0$ and so $\delta_{A}\left\{k: x_{k} \notin S_{\varepsilon}(C \backslash\{\zeta\})\right\}=0$. This shows that $C$ is not an $A$-minimal set, a contradiction.

Case 2. If $\zeta$ is a limit point of the set $C$ then there is a sequence $\left\{\zeta_{m}\right\}_{m \in \mathbb{N}}$ in $C$ such that $\left\{\zeta_{m}\right\}_{m \in \mathbb{N}}$ converges to $\zeta$ and $\zeta_{j} \neq \zeta_{i}$ for $i \neq j$. Let $\varepsilon>0$ be given. Choose $\zeta^{\prime}=\zeta_{m}$ such that $\left\|\zeta-\zeta^{\prime}\right\|=2 \delta$ with $4 \delta<\varepsilon$.

We claim that $S_{\delta}(C) \subset S_{\varepsilon}\left(C \backslash S_{\delta}(\zeta)\right)$. Let $x \in S_{\delta}(C)$ and $x^{\prime} \in C$ be such that $\left\|x-x^{\prime}\right\|<\delta$. If $x^{\prime} \notin S_{\delta}(\zeta)$ then $x^{\prime} \in$ $C \backslash S_{\delta}(\zeta)$ and so $x \in S_{\delta}\left(C \backslash S_{\delta}(\zeta)\right) \subset S_{\varepsilon}\left(C \backslash S_{\delta}(\zeta)\right)$. Again if $x^{\prime} \in S_{\delta}(\zeta)$ then $\|x-\zeta\| \leq\left\|x-x^{\prime}\right\|+\left\|x^{\prime}-\zeta\right\|+\left\|\zeta-\zeta^{\prime}\right\|<4 \delta<\varepsilon$.
But $\left\|\zeta-\zeta^{\prime}\right\|=2 \delta$ and so $\zeta^{\prime} \in C \backslash S_{\delta}(\zeta)$. Hence $x \in S_{\varepsilon}\left(C \backslash S_{\delta}(\zeta)\right)$ and so $S_{\delta}(C) \subset S_{\varepsilon}\left(C \backslash S_{\delta}(\zeta)\right)$ which implies that

$$
\left\{k: x_{k} \notin S_{\varepsilon}\left(C \backslash S_{\delta}(\zeta)\right)\right\} \subseteq\left\{k: x_{k} \notin S_{\delta}(C)\right\} .
$$

Hence, $\delta_{A}\left\{k: x_{k} \notin S_{\varepsilon}\left(C \backslash S_{\delta}(\zeta)\right)\right\}=0$ and so $C$ is not the $A$-minimal set. This completes the proof of the theorem.

\section{An Application to Turnpike Theorem}

We consider the following system:

$$
x_{k+1}=f\left(x_{k}, u_{k}\right), \quad x_{1}=\zeta^{0},
$$

where $\zeta^{0} \in \mathbb{R}^{m}$ is the assigned initial point, function $f(x, u)$ : $\mathbb{R}^{m} \times \mathbb{R}^{r} \rightarrow \mathbb{R}^{m}$ is continuous, $u \in U$, and $U \subset \mathbb{R}^{r}$ is a compact set. The sequences $x=\left\{x_{k}\right\}_{k \in \mathbb{N}}$ and $u=\left\{u_{k}\right\}_{k \in \mathbb{N}}\left(u_{k} \in\right.$ $U$, for every $k$ ) are called, a path and a control respectively, if (21) is satisfied for every $k \in \mathbb{N}$. The pair $(u, x)$ is called a process. We assume that there exists a bounded closed set $C \subset \mathbb{R}^{m}$ such that $x_{k} \in C$ for every path $x=\left\{x_{k}\right\}_{k \in \mathbb{N}}$ that is $\mathscr{I}$-bounded. The point $\zeta \in \mathbb{R}^{m}$ is called a stationary point if there exists $u \in U$ such that $f(\zeta, u)=\zeta$. We denote the set of all stationary points by $M$. It is clear that $M$ is a closed set. Let $\phi: \mathbb{R}^{m} \rightarrow \mathbb{R}$ be a given continuous function by which we shall define a functional.

Let $\alpha=\left(\alpha_{k}\right)_{k \in \mathbb{N}}$ be a $\mathscr{I}$-bounded number sequence and let $\Gamma_{\mathscr{I}}(\alpha)$ be the set of $\mathscr{I}$-cluster points of this sequence. We denote by $\mathscr{C}-\liminf _{k \rightarrow \infty} \alpha_{k}$ the minimal element in $\Gamma_{\mathscr{I}}(\alpha)$. If $x=\left\{x_{k}\right\}_{k \in \mathbb{N}}$ is a path to (21) then $\phi=\phi\left(x_{k}\right)$ is a $\mathscr{I}$-bounded number sequence. So on the paths to (21), we can consider the problem in line of [27]:

$$
J(x)=\mathscr{C}-\liminf _{k \rightarrow \infty} \phi\left(x_{k}\right) \longrightarrow \max .
$$

First we prove the following lemma.

Lemma 20. Let $\phi: \mathbb{R}^{m} \rightarrow \mathbb{R}$ be a continuous function. Then the functional $J(x)$ may be expressed in the form

$$
J(x)=\min _{\zeta \in \Gamma_{\mathcal{J}}(x)} \phi(\zeta) .
$$

Proof. Since the path $x=\left\{x_{k}\right\}_{k \in \mathbb{N}}$ is $\mathscr{I}$-bounded, so $\Gamma_{\mathscr{I}}(x)$ is nonempty and compact. Again since the function $\phi$ is continuous, so there exists a point $\zeta^{*} \in \Gamma_{\mathscr{I}}(x)$ such that $\phi^{*}=\phi\left(\zeta^{*}\right)=\min _{\zeta \in \Gamma_{\mathcal{F}}(x)} \phi(\zeta)$.

Let $\Gamma_{\mathscr{I}}(\phi)$ denote the set of $\mathscr{I}$-cluster points of the sequence $\left\{\phi\left(x_{k}\right)\right\}_{k \in \mathbb{N}}$. Now we will show that $\min \Gamma_{\mathscr{I}}(\phi)=\phi^{*}$. Since $\zeta^{*} \in \Gamma_{\mathscr{J}}(x)$, for every $\delta>0,\left\{n \in \mathbb{N}:\left\|x_{k}-\zeta^{*}\right\|<\delta\right\} \notin$ $\mathscr{I}$. Let $\varepsilon>0$ be given. Then there exists $\delta=\delta(\varepsilon)>0$ such that $\left|\phi(\zeta)-\phi\left(\zeta^{*}\right)\right|<\varepsilon$ for every $\zeta,\left\|\zeta-\zeta^{*}\right\|<\delta$. Therefore $\left\{k \in \mathbb{N}:\left|\phi\left(x_{k}\right)-\phi\left(\zeta^{*}\right)\right|<\varepsilon\right\} \supset\left\{k \in \mathbb{N}:\left\|x_{k}-\zeta^{*}\right\|<\delta\right\}$ which implies that $\left\{k \in \mathbb{N}:\left|\phi\left(x_{k}\right)-\phi\left(\zeta^{*}\right)\right|<\varepsilon\right\} \notin \mathscr{I}$ and so $\left\{k \in \mathbb{N}:\left|\phi\left(x_{k}\right)-\phi^{*}\right|<\mathcal{E}\right\} \notin \mathscr{I}$ that is, $\phi^{*} \in \Gamma_{\mathscr{I}}(\phi)$. Hence $\min \Gamma_{\mathscr{I}}(\phi) \leq \phi^{*}$.

Now assume that $\phi^{\prime} \in \Gamma_{\mathscr{J}}(\phi)$ and $\phi^{\prime} \leq \phi^{*}$. Let $\varepsilon=$ $\left(\phi^{*}-\phi^{\prime}\right) / 2$. Since $\phi$ is continuous, there exists $\delta>0$ such that $\min _{\zeta \in S_{\delta}\left(\Gamma_{\mathscr{J}}(x)\right)} \phi(\zeta) \geq \phi^{*}-\varepsilon$. Put $B=\left\{k \in \mathbb{N}: d\left(\Gamma_{\mathscr{I}}(x), x_{k}\right) \geq\right.$ $\delta$. Obviously $B \in \mathscr{I}$. Then $d\left(\Gamma_{\mathscr{I}}(x), x_{k}\right)<\delta$ forall $\mathbb{N} \backslash \mathrm{B}$, 
that is, $x_{k} \in S_{\delta}\left(\Gamma_{\mathscr{F}}(x)\right)$ and so $\phi\left(x_{k}\right) \geq \phi^{*}-\varepsilon$ forall $\mathrm{k} \in \mathbb{N} \backslash \mathrm{B}$. Again $\varepsilon=\left(\phi^{*}-\phi^{\prime}\right) / 2$ implies $\phi^{*}-\varepsilon=\phi^{\prime}+\varepsilon$. Therefore $\phi\left(x_{k}\right) \geq \phi^{\prime}-\varepsilon$ forall $\mathrm{k} \in \mathbb{N} \backslash \mathrm{B}$. This shows that $\left|\phi\left(x_{k}\right)-\phi^{\prime}\right|<\varepsilon$ can not be satisfied for $k \in \mathbb{N} \backslash B$. Hence $\left\{k \in \mathbb{N}: \mid \phi\left(x_{k}\right)-\right.$ $\left.\phi^{\prime} \mid<\varepsilon\right\} \subset B \in \mathscr{I}$ which implies that $\phi^{\prime} \notin \Gamma_{\mathscr{F}}(x)$. Hence $\min \Gamma_{\mathscr{J}}(\phi) \geq \phi^{*}$. Therefore $\min \Gamma_{\mathscr{I}}(\phi)=\phi^{*}$.

Now we will establish a general version of the Turnpike Theorem (see [27, 29-33] for more details and history) which turns out to be valid for a special class of ideals, namely those ideals which are invariant under translation.

Before we proceed further, we recall the following basic facts about analytic $P$-ideals (see $[34,35]$ ).

A map $\psi: P(\mathbb{N}) \rightarrow[0, \infty]$ is a submeasure on $\mathbb{N}$ if

(i) $\psi(\emptyset)=0$,

(ii) $\psi(A) \leq \psi(A \cup B) \leq \psi(A)+\psi(B)$, for all $A, B \subset \mathbb{N}$.

It is lower semicontinuous if for all $A \subset \mathbb{N}$, we have $\psi(A)=$ $\lim _{n \rightarrow \infty} \psi(A \backslash n)$, where $A \backslash n=A \backslash\{1,2,3, \ldots, n\}$. For every lower semicontinuous submeasure on $\mathbb{N}$, let $\|\cdot\|_{\psi}: P(\mathbb{N}) \rightarrow$ $[0, \infty]$ be the submeasure defined by

$$
\begin{aligned}
\|A\|_{\psi} & =\limsup _{n \rightarrow \infty} \psi(A \backslash n) \\
& =\lim _{n \rightarrow \infty} \psi(A \backslash n) .
\end{aligned}
$$

Let $\mathscr{I}=\operatorname{Exh}(\psi)=\left\{A \subset \mathbb{N}:\|A\|_{\psi}=0\right\}$. It is clear that $\operatorname{Exh}(\psi)$ is an ideal for an arbitrary submeasure $\psi$. An ideal $\mathscr{I}$ on $\mathbb{N}$ is an analytic $P$-ideal if $\mathscr{I}=\operatorname{Exh}(\psi)$ for some lower semicontinuous submeasure $\psi$ on $\mathbb{N}$. Following [35], we say that an ideal $\mathscr{I}$ is invariant under translation if for each $A \in$ $\mathscr{I}$, and $n \in \mathbb{Z}, A+n \in \mathscr{I}$ where $A+n=\{a+n: a \in A\} \cap \mathbb{N}$. If $\|\cdot\|_{\psi}$ is invariant under translations, then the ideal $\operatorname{Exh}(\psi)$ is invariant under translations.

Let $f: \mathbb{N} \rightarrow[0, \infty)$ be such that $\sum_{i=0}^{\infty} f(i)=$ $+\infty$ and $\lim _{n \rightarrow \infty}\left(f(n) / \sum_{i \in n} f(i)\right)=0$. Then $\varepsilon \mho_{f}=$ $\left\{A \subset \mathbb{N}: \lim _{n \rightarrow \infty}\left(\sum_{i \in A \cap n} f(i) / \sum_{i \in n} f(i)\right)=0\right\}$ is called an Erdös-Ulam ideal [35]. The ideal $\mathscr{I}_{d}$ consisting of all sets with statistical density zero is an Erdös-Ulam ideal (generated by any constant positive function $f$ ). Every Erdös-Ulam ideal $\varepsilon \mho_{f}$ is an analytic $P$-ideal of the form $\operatorname{Exh}\left(\psi_{f}\right)$, where $\psi_{f}(A)=\sup _{n \in \mathbb{N}}\left(\sum_{i \in A \cap n} f(i) / \sum_{i \in n} f(i)\right)$ and $\|A\|_{\psi_{f}}=\limsup _{n \rightarrow \infty}\left(\sum_{i \in A \cap n} f(i) / \sum_{i \in n} f(i)\right)$. Observe that if $\lim _{n \rightarrow \infty}\left(\sum_{i \in n}|f(i+k)-f(i)| / \sum_{i \in n} f(i)\right)=0$ for every $k \in \mathbb{Z}$ then $\|\cdot\|_{\psi_{f}}$ is invariant under translations. So ErdösUlam ideals are examples of ideals which are invariant under translation under certain conditions.

Following the line of [27] we now study the asymptotic stability of optimal paths in the problem (21) and (22) and for this we consider the main conditions as follows:

Condition 1 (in short $\mathrm{C} 1$ ). The maximum of the function $\phi$ on $M$ is reached at the unique stationary point $\zeta^{*}$ such that $\max _{\zeta \in M} \phi(\zeta)=\phi\left(\zeta^{*}\right)$.
Condition 2 (in short C2). There exists a process $\left(u^{*}, x^{*}\right)$ such that $x_{k}{ }^{*} \rightarrow \zeta^{*}$ as $k \rightarrow \infty$.

Condition 3 (in short C3). There exists a vector $p \in \mathbb{R}^{m},\|p\|=$ 1 such that $p f(x, u)<p x$ for every $x \in B, x \neq \zeta^{*}$ and $u \in U$ where $B=\left\{\zeta \in C: \phi(\zeta) \geq \phi\left(\zeta^{*}\right)\right\}$.

Theorem 21 (Turnpike Theorem). Let conditions C1, C2, and $C 3$ hold and let $(u, x)$ be an optimal process in the problems (21) and (22). Then has $\Gamma_{\mathscr{I}}(x)=\left\{\zeta^{*}\right\}$, where the ideal $\mathscr{I}$ is invariant under translation.

Proof. By C2 for the process $\left(u^{*}, x^{*}\right)=\left(u_{k}^{*}, x_{k}^{*}\right)$ we have $\Gamma_{\mathscr{I}}\left(x^{*}\right)=\left\{\zeta^{*}\right\}$. Hence $J\left(x^{*}\right)=\min _{\zeta \in \Gamma_{\mathscr{I}}\left(x^{*}\right)} \phi(\zeta)=\phi\left(\zeta^{*}\right)$ from Lemma 20. Therefore the maximal quantity of the functional (22) is not less than $\phi\left(\zeta^{*}\right)$. As $(u, x)$ is an optimal process, so $J(x)=\min _{\zeta \in \Gamma_{\mathcal{F}}(x)} \phi(\zeta) \geq \phi\left(\zeta^{*}\right)$. Thus $\Gamma_{\mathscr{I}}(x) \subset B$.

Define the function $F(\zeta)=\max _{u \in U} p f(\zeta, u)-p \zeta$. It is clear that $F(\zeta)$ is a continuous function. From C3 it follows that for every $\zeta \in \Gamma_{\mathscr{I}}(x), \zeta \neq \zeta^{*}$, the inequality $F(\zeta)<0$ is satisfied and at the point $\zeta^{*}$ we have $F\left(\zeta^{*}\right)=0$.

Claim. $\Gamma_{\mathscr{I}}(x)=\left\{\zeta^{*}\right\}$

Case 1. Suppose that there is a point $\zeta_{1} \in \Gamma_{\mathscr{F}}(x)$ such that $\zeta_{1} \neq \zeta^{*}$ and $\min _{\zeta \in \Gamma_{\mathcal{f}}(x)} p \zeta=p \zeta_{1}$. We have $F\left(\zeta_{1}\right)<0$. Since $F(\zeta)$ is continuous, so there exists a number $\gamma>0$ such that

$$
F(\zeta)<-4 \gamma \text { for every } \zeta \in S_{\gamma}\left(\zeta_{1}\right)
$$

where $S_{\gamma}(A)=\left\{\zeta \in \mathbb{R}^{m}: d(\zeta, A) \leq \gamma\right\}$.

Let $x_{k} \in S_{\gamma}\left(\zeta_{1}\right)$. Then from (25), $F\left(x_{k}\right)<-4 \gamma$ or $p f\left(x_{k}, u\right)-p x_{k}<-4 \gamma$ for every $u \in U$. In particular for the point $u_{k} \in U$ we have $p x_{k+1}=p f\left(x_{k}, u_{k}\right)<p x_{k}-4 \gamma$. As $\|p\|=1$ and $x_{k} \in S_{\gamma}\left(\zeta_{1}\right)$, so we obtain $p x_{k} \leq p \zeta_{1}+\gamma$. Thus $p x_{k+1}<p \zeta_{1}-3 \gamma$. On the other hand for every point $\zeta \epsilon$ $S_{\gamma}\left(\Gamma_{\mathscr{I}}(x)\right), p \zeta \geq \min _{y \in \Gamma_{\mathscr{J}}(x)} p y-\gamma=p \zeta_{1}-\gamma>p \zeta_{1}-3 \gamma$. Then $x_{k+1} \notin S_{\gamma}\left(\Gamma_{\mathscr{J}}(x)\right)$. Thus if $x_{k} \in S_{\gamma}\left(\zeta_{1}\right)$ then $x_{k+1} \notin S_{\gamma}\left(\Gamma_{\mathscr{I}}(x)\right)$.

Let $K_{\gamma}=\left\{k \in \mathbb{N}:\left\|x_{k}-\zeta_{1}\right\|<\gamma\right\}=\left\{k_{1}, k_{2}, k_{3}, \ldots\right\}$, $Z=\left\{k \in \mathbb{N}: d\left(\Gamma_{\mathscr{I}}(x), x_{k}\right) \geq \gamma\right\}$ and consider the set $A=$ $\left\{k_{1}+1, k_{2}+1, k_{3}+1, \ldots\right\}$. As $\zeta_{1}$ is a $\mathscr{I}$-cluster point so $K_{\gamma} \notin \mathscr{I}$. Since $\mathscr{I}$ is assumed to be invariant under translation so we have $A \notin \mathscr{I}$. But $A \subset Z$ and so $Z \notin \mathscr{I}$, a contradiction. Thus $\zeta^{*}$ is a unique $\mathscr{I}$-cluster point for which $p \zeta^{*}=\min _{\zeta \in \Gamma_{\mathscr{f}}(x)} p \zeta$, that is; $p \zeta^{*}<p \zeta$ for every $\zeta \in \Gamma_{\mathscr{I}}(x), \zeta \neq \zeta^{*}$.

Case 2. Let now $\zeta_{2} \in \Gamma_{\mathscr{I}}(x)$ be such that $\zeta_{2} \neq \zeta^{*}$ and $\max _{\zeta \in \Gamma_{\mathcal{J}}(x)} p \zeta=p \zeta_{2}$. Let $p \zeta_{2}-p \zeta^{*}=4 a(a>0)$. Now C1 implies that $F\left(\zeta^{*}\right)=0$. As $F(\zeta)$ is continuous, for the number $a$ we can find a number $0<\delta<a$ such that $F(\zeta)<a$ for every $\zeta \in S_{\delta}\left(\zeta^{*}\right)$.

Let $x_{k} \in S_{\delta}\left(\zeta^{*}\right)$. Then $F\left(x_{k}\right)<a$ or $p f\left(x_{k}, u\right)-p x_{k}<a$ for every $u \in U$. In particular for the point $u_{k} \in U, p x_{k+1}<p x_{k}+$ a. But $p x_{k} \leq p \zeta^{*}+\delta<p \zeta^{*}+a$. Therefore $p x_{k+1}<p \zeta^{*}+2 a$. On the other hand for every point $\zeta \in S_{a}\left(\zeta_{2}\right)$ we can write $p \zeta \geq p \zeta_{2}-a=\left(p \zeta^{*}+4 a\right)-a=p \zeta^{*}+3 a>p \zeta^{*}+2 a$. Thus $x_{k+1} \notin S_{a}\left(\zeta_{2}\right)$ if $x_{k} \in S_{\delta}\left(\zeta^{*}\right)$. 
Consider the set $\Delta_{1}=\left\{\zeta \in \Gamma_{\mathcal{F}}(x):\left\|\zeta-\zeta^{*}\right\| \leq \delta / 2\right\}$. Obviously $S_{\delta / 2}\left(\Delta_{1}\right) \subset S_{\delta}\left(\zeta^{*}\right)$ and therefore we obtain that

$$
\text { if } x_{k} \in S_{\delta / 2}\left(\Delta_{1}\right) \text { then } x_{k+1} \notin S_{a}\left(\zeta_{2}\right) \text {. }
$$

Again if $\Delta_{2}=\left\{\zeta \in \Gamma_{\mathscr{I}}(x):\left\|\zeta-\zeta^{*}\right\| \geq \delta / 2\right\}$, then it is clear that $\Delta_{2}$ is compact, $\Delta_{2} \subset B$ and $\zeta^{*} \notin \Delta_{2}$. By C3, we have $F(\zeta)<0$ for every $\zeta \in \Delta_{2}$. As $F(x)$ is continuous there exists a number $\gamma>0$ such that $F(\zeta)<-4 \gamma$ for every $\zeta \in S_{\gamma}\left(\Delta_{2}\right)$.

Let $x_{k} \in S_{\gamma}\left(\Delta_{2}\right)$. Then $F\left(x_{k}\right)=\max _{u \in U} p f\left(x_{k}, u\right)-p x_{k}<$ $-4 \gamma$ and in particular for the point $u_{k} \in U, p x_{k+1}<p x_{k}-4 \gamma$. It is clear that $p x_{k} \leq \max _{\zeta \in S_{\delta}\left(\Delta_{2}\right)} p \zeta+\gamma=p \zeta_{2}+\gamma$. Therefore $p x_{k+1}<p \zeta_{2}-3 \gamma$. On the other hand for every $\zeta \in S_{\gamma}\left(\zeta_{2}\right)$, $p \zeta \geq p \zeta_{2}-\gamma>p \zeta_{2}-3 \gamma$. Then $x_{k+1} \notin S_{\gamma}\left(\zeta_{2}\right)$. Thus we obtain that

$$
\text { if } x_{k} \in S_{\gamma}\left(\Delta_{2}\right) \text { then } x_{k+1} \notin S_{\gamma}\left(\zeta_{2}\right) \text {. }
$$

Now let $\varepsilon=\min (\delta / 2, a, \gamma)$. It is clear that $\Gamma_{\mathscr{f}}(x)=\Delta_{1} \cup \Delta_{2}$ and therefore $S_{\varepsilon}\left(\Gamma_{\mathcal{J}}(x)\right)=S_{\varepsilon}\left(\Delta_{1}\right) \cup S_{\varepsilon}\left(\Delta_{2}\right)$. Then from (26) and (27) it follows if $x_{k} \in S_{\varepsilon}\left(\Gamma_{\mathscr{F}}(x)\right)$, then $x_{k+1} \in S_{\varepsilon}\left(\zeta_{2}\right)$ which implies that if $x_{k} \in S_{\varepsilon}\left(\zeta_{2}\right)$, then $x_{k-1} \notin S_{\varepsilon}\left(\Gamma_{\mathscr{F}}(x)\right)$.

Let $K_{\varepsilon}:=\left\{k \in \mathbb{N}:\left\|x_{k}-\zeta_{2}\right\|<\varepsilon\right\}=\left\{k_{1}, k_{2}, k_{3}, \ldots\right\}, Z=$ $\left\{k \in \mathbb{N}: d\left(\Gamma_{\mathscr{F}}(x), x_{k}\right) \geq \varepsilon\right\}$ and $A=\left\{k_{1}-1, k_{2}-1, k_{3}-1, \ldots\right\}$. As $\zeta_{2}$ is a $\mathscr{I}$-cluster point so $K_{\varepsilon} \notin \mathscr{I}$ which consequently implies $A \notin \mathscr{I}$. Again $A \subset Z$ and so $Z \notin \mathscr{I}$, a contradiction. Thus $\Gamma_{\mathscr{J}}(x)=\left\{\zeta^{*}\right\}$ that is, the path $\left\{x_{k}\right\}_{k \in \mathbb{N}}$ is $\mathscr{J}_{\text {-convergent }}$ to $\zeta^{*}$.

Remark 22. We consider the system

$$
x_{k+1} \in x_{k}+a\left(x_{k}\right), x_{1}=\zeta^{0}, k \in \mathbb{N},
$$

where $\zeta^{0} \in \mathbb{R}^{m}$ is a given initial point and $a: \mathbb{R}^{m} \rightarrow$ $\mathbb{R}^{m}$ is a multivalued mapping having compact images and is continuous in the Hausdorff metric.

Now, following the line of Mamedov and Pehlivan [32], we can show the following.

Theorem 23 (Turnpike Theorem). Let conditions C1, C2, and C3 hold and let $x=\left\{x_{k}\right\}_{k \in \mathbb{N}}$ be an asymptotical optimal path satisfying the conditions of the systems (28) and (22). Then has $\Gamma_{\mathscr{f}}(x)=\left\{\zeta^{*}\right\}$, where the ideal $\mathscr{I}$ is invariant under translation.

\section{Conflict of Interests}

The authors declare that there is no conflict of interests regarding the publication of this paper.

\section{Acknowledgments}

The authors gratefully acknowledge the financial support from King Abdulaziz University, Jeddah, Saudi Arabia. The first author is thankful to CSIR for granting Project no. 25(0186)/10/EMR-II during the tenure of which this work was done. The second author is thankful to CSIR, India, for giving JRF during the tenure of which this work was done.

\section{References}

[1] E. Kolk, "The statistical convergence in Banach spaces," Acta et Commentationes Universitatis Tartuensis, no. 928, pp. 41-52, 1991.

[2] H. Fast, "Sur la convergence statistique," vol. 2, pp. 241-244, 1951.

[3] J. A. Fridy, "On statistical convergence," Analysis, vol. 5, no. 4, pp. 301-313, 1985.

[4] J. A. Fridy, "Statistical limit points," Proceedings of the American Mathematical Society, vol. 118, no. 4, pp. 1187-1192, 1993.

[5] J. S. Connor, "The statistical and strong $p$-Cesàro convergence of sequences," Analysis, vol. 8, no. 1-2, pp. 47-63, 1988.

[6] T. Šalát, "On statistically convergent sequences of real numbers," Mathematica Slovaca, vol. 30, no. 2, pp. 139-150, 1980.

[7] C. Belen and S. A. Mohiuddine, "Generalized weighted statistical convergence and application," Applied Mathematics and Computation, vol. 219, no. 18, pp. 9821-9826, 2013.

[8] J. Činčura, T. Šalát, M. Sleziak, and V. Toma, "Sets of statistical cluster points and $\mathscr{I}$-cluster points," Real Analysis Exchange, vol. 30, no. 2, pp. 565-580, 2004-2005.

[9] B. K. Lahiri and P. Das, " $\mathscr{I}$ and $\mathscr{I}^{*}$-convergence in topological spaces," Mathematica Bohemica, vol. 130, no. 2, pp. 153-160, 2005.

[10] B. K. Lahiri and P. Das, “ $\mathscr{I}$ and $\mathscr{I}^{*}$-convergence of nets," Real Analysis Exchange, vol. 33, no. 2, pp. 431-442, 2008.

[11] S. A. Mohiuddine, A. Alotaibi, and M. Mursaleen, "A new variant of statistical convergence," Journal of Inequalities and Applications, vol. 2013, article 309, 8 pages, 2013.

[12] P. Kostyrko, T. Šalát, and W. Wilczyński, “ $\mathscr{I}$-convergence," Real Analysis Exchange, vol. 26, no. 2, pp. 669-685, 2000/01.

[13] J. Connor and J. Kline, "On statistical limit points and the consistency of statistical convergence," Journal of Mathematical Analysis and Applications, vol. 197, no. 2, pp. 392-399, 1996.

[14] P. Das and S. Ghosal, "When $\mathscr{I}$-Cauchy nets in complete uniform spaces are $\mathscr{I}$-convergent," Topology and Its Applications, vol. 158, no. 13, pp. 1529-1533, 2011.

[15] P. Das and S. K. Ghosal, "On $\mathscr{I}$-Cauchy nets and completeness," Topology and Its Applications, vol. 157, no. 7, pp. 1152-1156, 2010.

[16] P. Das, E. Savas, and S. K. Ghosal, "On generalizations of certain summability methods using ideals," Applied Mathematics Letters, vol. 24, no. 9, pp. 1509-1514, 2011.

[17] L. W. McKenzie, “Turnpike theory," Econometrica, vol. 44, no. 5, pp. 841-865, 1976.

[18] S. A. Mohiuddine, A. Alotaibi, and S. M. Alsulami, "Ideal convergence of double sequences in random 2-normed spaces," Advances in Difference Equations, vol. 2012, article 149, 8 pages, 2012.

[19] M. Mursaleen and S. A. Mohiuddine, "On ideal convergence in probabilistic normed spaces," Mathematica Slovaca, vol. 62, no. 1, pp. 49-62, 2012.

[20] M. Mursaleen and S. A. Mohiuddine, "On ideal convergence of double sequences in probabilistic normed spaces," Mathematical Reports, vol. 12 (62), no. 4, pp. 359-371, 2010.

[21] E. Savas, P. Das, and S. Dutta, "A note on strong matrix summability via ideals," Applied Mathematics Letters, vol. 25, no. 4, pp. 733-738, 2012.

[22] K. Demirci, "Strong $A$-summability and $A$-statistical convergence," Indian Journal of Pure and Applied Mathematics, vol. 27, no. 6, pp. 589-593, 1996.

[23] K. Demirci, “A-statistical core of a sequence," Demonstratio Mathematica, vol. 33, no. 2, pp. 343-353, 2000. 
[24] S. Pehlivan, H. Albayrak, and H. Z. Toyganözü, "The theory of convergence and the set of statistical cluster points," Advances in Dynamical Systems and Applications, vol. 6, no. 1, pp. 111-119, 2011.

[25] P. Letavaj, “ $\mathscr{I}$-convergence to a set," Acta Mathematica Universitatis Comenianae, vol. 80, no. 1, pp. 103-106, 2011.

[26] S. Pehlivan, A. Güncan, and M. A. Mamedov, "Statistical cluster points of sequences in finite dimensional spaces," Czechoslovak Mathematical Journal, vol. 54, no. 1, pp. 95-102, 2004.

[27] M. A. Mamedov and S. Pehlivan, "Statistical convergence of optimal paths," Mathematica Japonica, vol. 52, no. 1, pp. 51-55, 2000.

[28] S. Pehlivan and M. A. Mamedov, "Statistical cluster points and turnpike," Optimization, vol. 48, no. 1, pp. 93-106, 2000.

[29] A. N. Lyapunov, "Asymptotical optimal paths for convex mappings," in Optimal Models in System Analysis, vol. 9, pp. 74-80, VNIISI, Moscow, Russia, 1983.

[30] V. L. Makarov, M. J. Levin, and A. M. Rubinov, Mathematical Economic Theory: Pure and Mixed Types of Economic Mechanisms, vol. 33 of Advanced Textbooks in Economics, NorthHolland, Amsterdam, The Netherlands, 1995.

[31] M. A. Mamedov, "Turnpike theorems in continuous systems with integral functionals," Doklady Akademii Nauk, vol. 323, no. 5, pp. 830-833, 1992, English translation: Russian Academy of Sciences. Doklady Mathematics, vol. 45, no. 2, 1992.

[32] M. A. Mamedov and S. Pehlivan, "Statistical cluster points and turnpike theorem in nonconvex problems," Journal of Mathematical Analysis and Applications, vol. 256, no. 2, pp. 686693, 2001.

[33] A. J. Zaslavski, "Turnpike theorem for a class of differential inclusions arising in economic dynamics," Optimization, vol. 42, no. 2, pp. 139-168, 1997.

[34] N. Mrożek, "Ideal version of Egorov's theorem for analytic $P$ ideals," Journal of Mathematical Analysis and Applications, vol. 349, no. 2, pp. 452-458, 2009.

[35] R. Filipów and P. Szuca, "Density versions of Schur's theorem for ideals generated by submeasures," Journal of Combinatorial Theory A, vol. 117, no. 7, pp. 943-956, 2010. 


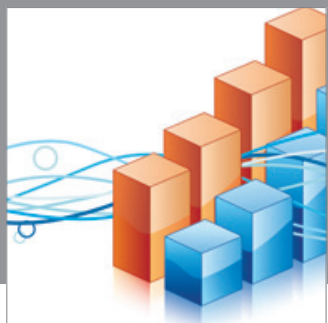

Advances in

Operations Research

mansans

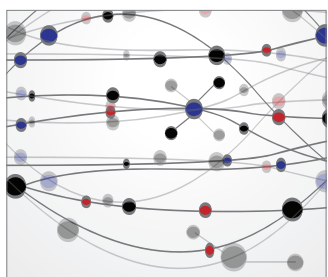

The Scientific World Journal
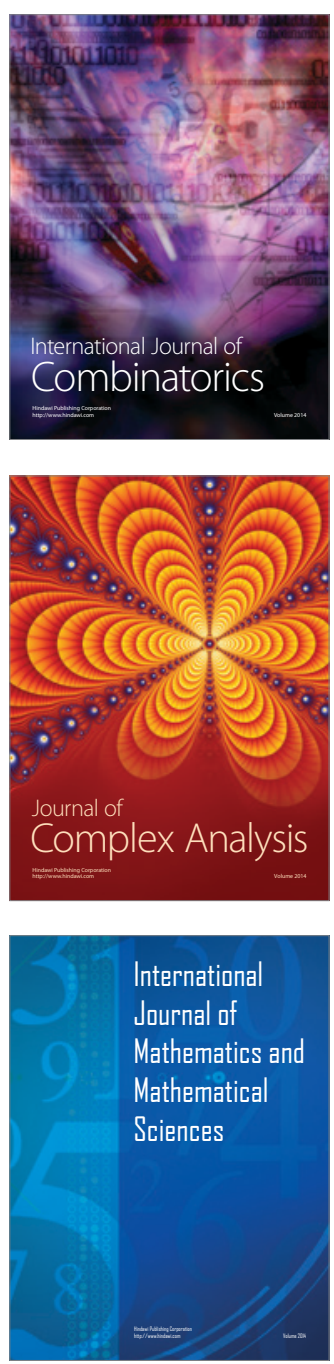
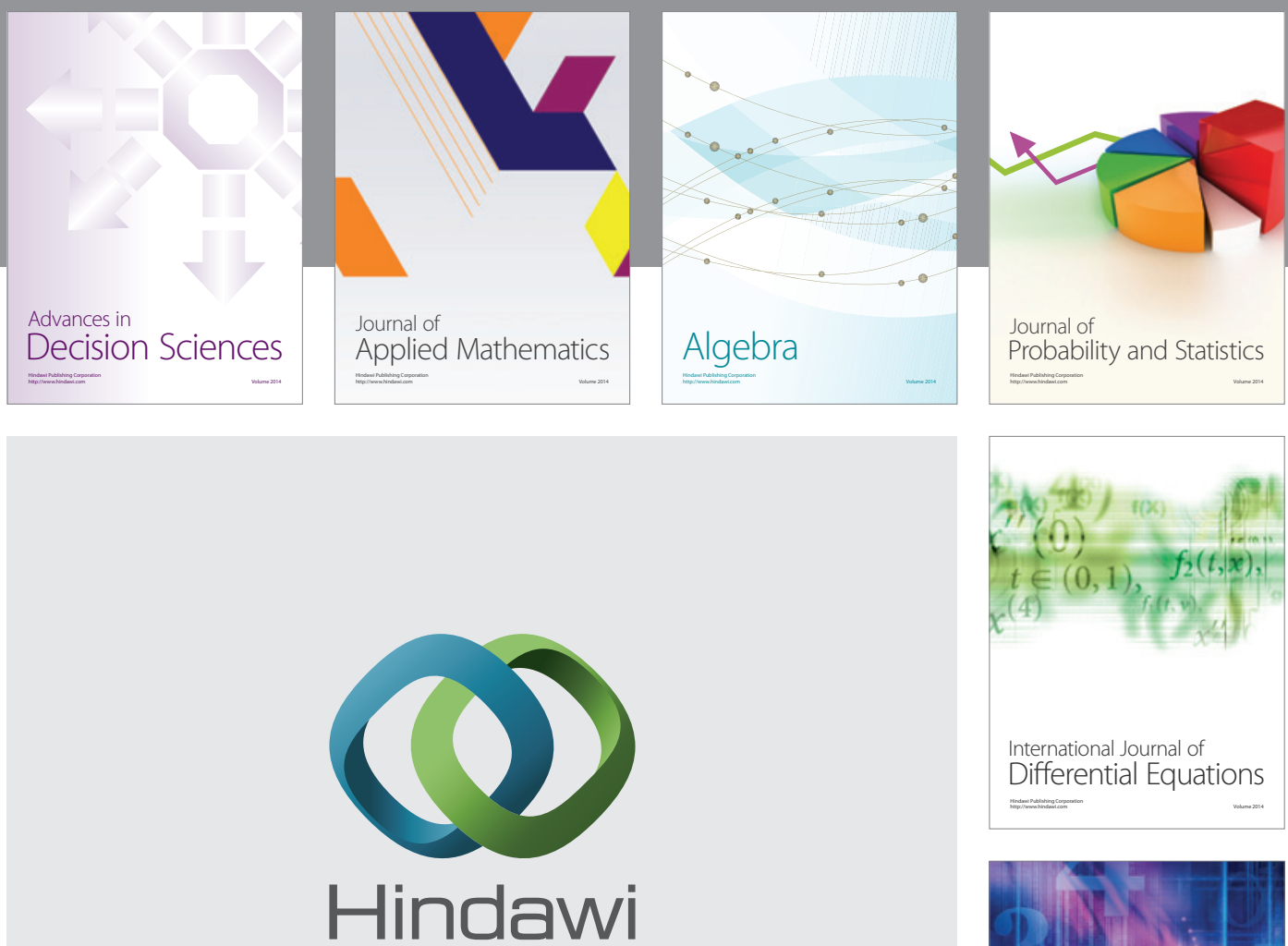

Submit your manuscripts at http://www.hindawi.com
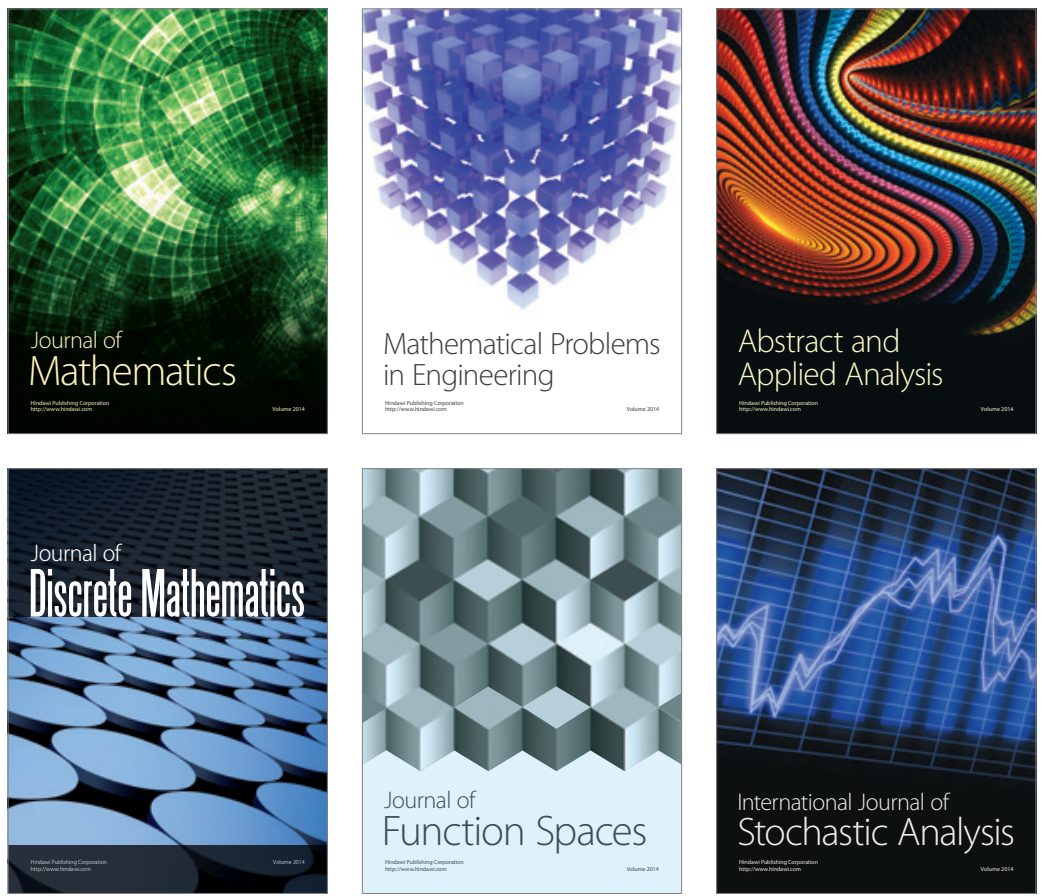

Journal of

Function Spaces

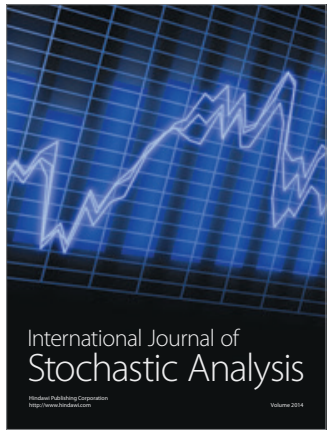

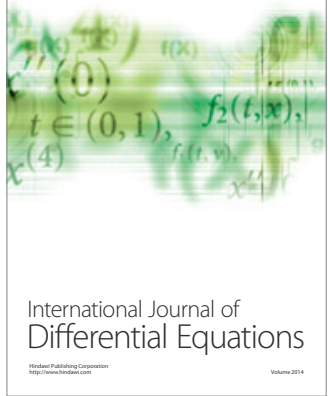
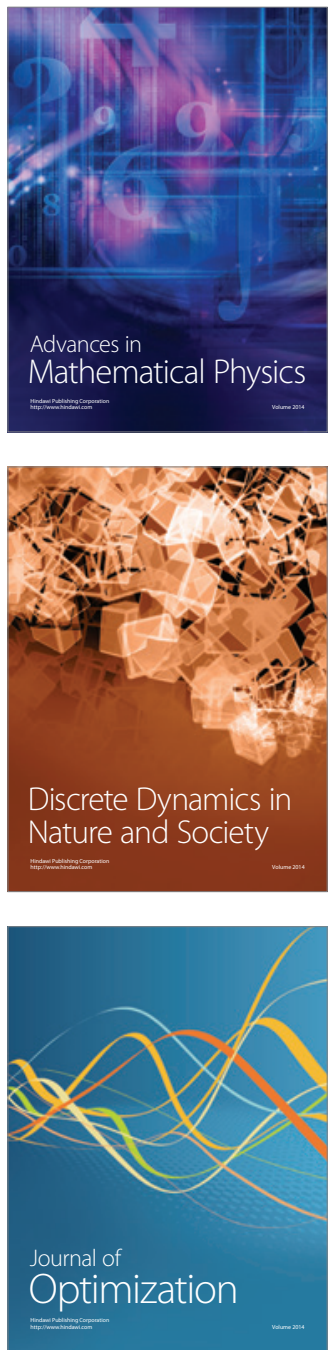